\title{
Síndrome Inflamatória Multissistêmica Pediátrica (SIM-P) temporariamente associada à COVID-19: um levantamento das características clínicas e epidemiológicas
}

\author{
Pediatric Multisystemic Inflammatory Syndrome (MIS-P) temporarily associated with COVID-19: \\ a survey of clinical and epidemiological characteristics \\ Síndrome Inflamatorio Multisistémico Pediátrico (SIM-P) asociado temporalmente a COVID-19:
} una encuesta de características clínicas y epidemiológicas

Bruno Wesley Ramalho Cirilo Ferreira ORCID: https://orcid.org/0000-0003-3448-0957 Residência Multiprofissional em Saúde da Criança, Brasil E-mail: brunnoramallho@hotmail.com

Anais Bezerra de Gusmão

ORCID: https://orcid.org/0000-0001-7170-9410 Residência Multiprofissional em Saúde da Criança, Brasil E-mail: anaisgusmao@gmail.com

Pollyana Amorim Ponce de Leon ORCID: https://orcid.org/0000-0003-4291-9536 Residência Multiprofissional em Saúde da Criança, Brasil E-mail: pollyanaleon@yahoo.com.br

Rossana Karla Gois Ferreira ORCID: https://orcid.org/0000-0001-8541-7929 Residência Multiprofissional em Saúde da Criança, Brasil E-mail: rossanagoisf@gmail.com Cibério Landim Macedo ORCID: https://orcid.org/0000-0002-0824-4056 Residência Multiprofissional em Saúde da Criança, Brasil E-mail: ciberiolandim@hotmail.com

\begin{abstract}
Resumo
O objetivo do presente estudo foi analisar a literatura científica atual a respeito da Síndrome Inflamatória Multissistêmica Pediátrica (SIM-P) temporariamente associada à COVID-19, a fim de documentar os principais achados e seu manejo terapêutico. Realizou-se uma revisão sistemática de estudos nas bases de dados US National Library of Medicine (PubMed), Scientific Electronic Library Online (Scielo) e ScienceDirect, entre os meses de setembro de 2020 a fevereiro de 2021, utilizando os descritores multisystem inflammatory syndrome, children e COVID-19. Os artigos incluídos apresentaram estudos observacionais com pacientes diagnosticados com SIM-P, artigos originais e metanálises publicados entre os anos de 2020 e 2021. Foram excluídos os estudos que não descreviam suficientemente os dados, que não apresentavam relação com objetivo tema desta revisão, bem como notícias, comentários, cartas de apresentação e duplicatas de artigos. Dos 668 estudos encontrados, 27 compuseram esta revisão. A SIM-P acomete crianças e adolescentes entre 0 a 19 anos, apresentando febre persistente, sintomas gastrointestinais, falta de ar, dor abdominal e disfunções orgânicas. Essa síndrome é uma resposta imunológica inflamatória retardada à infecção recente por SARS-CoV-2, exibindo alterações nos marcadores inflamatórios e de outros indicadores, associados a alterações nos exames de imagem. O manejo terapêutico visa reduzir a resposta inflamatória sistêmica e reestabelecimento das funções orgânicas utilizando imunoglobulina, corticosteroides, drogas vasoativas, imunomoduladores e anticoagulantes. Poucas evidências científicas estão disponíveis para entender essa síndrome. Assim, estudos multicêntricos e prospectivos são necessários para melhor compreensão da fisiopatologia, critérios diagnósticos, tratamento e existência de complicações de médio a longo prazo.
\end{abstract}

Palavras-chave: Infecções por coronavírus; Inflamação; Pediatria.

\footnotetext{
Abstract

The aim of the present study was to analyze the current scientific literature regarding Pediatric Multisystemic Inflammatory Syndrome (SIM-P) temporarily associated with COVID-19, in order to document the main findings and their therapeutic management. A systematic review of studies was carried out in the databases US National Library of
} 
Medicine (PubMed), Scientific Electronic Library Online (Scielo) and ScienceDirect, from September 2020 to February 2021, using the descriptors multisystem inflammatory syndrome, children and COVID-19. The included articles presented observational studies with patients diagnosed with SIM-P, original articles and meta-analyzes published between the years 2020 and 2021. Studies that did not sufficiently describe the data, which were not related to the objective of this review, as well as news, comments, cover letters and duplicates of articles were excluded. Of the 668 studies found, 27 comprised this review. SIM-P affects children and adolescents between 0 and 19 years old, with persistent fever, gastrointestinal symptoms, shortness of breath, abdominal pain and organ dysfunctions. This syndrome is a delayed inflammatory immune response to recent SARS-CoV-2 infection, showing changes in inflammatory markers and other indicators, associated with changes in imaging tests. Therapeutic management aims to reduce the systemic inflammatory response and reestablish organic functions using immunoglobulin, corticosteroids, vasoactive drugs, immunomodulators and anticoagulants. Little scientific evidence is available to understand this syndrome. Thus, multicenter and prospective studies are necessary to better understand the pathophysiology, diagnostic criteria, treatment and existence of medium to long term complications.

Keywords: Coronavirus infections; Inflammation; Pediatrics.

\section{Resumen}

El objetivo del presente estudio fue analizar la literatura científica actual sobre el Síndrome Inflamatorio Multisistémico Pediátrico (SIM-P) asociado temporalmente al COVID-19, con el fin de documentar los principales hallazgos y su manejo terapéutico. Se realizó una revisión sistemática de estudios en las bases de datos US National Library of Medicine (PubMed), Scientific Electronic Library Online (Scielo) y ScienceDirect, de septiembre de 2020 a febrero de 2021, utilizando los descriptores síndrome inflamatorio multisistémico, niños y COVID-19. Los artículos incluidos presentaban estudios observacionales con pacientes diagnosticados de SIM-P, artículos originales y metaanálisis publicados entre los años 2020 y 2021. Se excluyeron los estudios que no describieron suficientemente los datos, que no estaban relacionados con el objetivo de esta revisión, así como noticias, comentarios, cartas de presentación y duplicados de artículos. De los 668 estudios encontrados, 27 incluyeron esta revisión. SIM-P afecta a niños y adolescentes entre 0 y 19 años, con fiebre persistente, síntomas gastrointestinales, disnea, dolor abdominal y disfunciones orgánicas. Este síndrome es una respuesta inmune inflamatoria retardada a una infección reciente por SARS-CoV-2, que muestra cambios en los marcadores inflamatorios y otros indicadores, asociados con cambios en las pruebas de imagen. El manejo terapéutico tiene como objetivo reducir la respuesta inflamatoria sistémica y restablecer las funciones orgánicas utilizando inmunoglobulinas, corticosteroides, fármacos vasoactivos, inmunomoduladores y anticoagulantes. Hay poca evidencia científica disponible para comprender este síndrome. Por tanto, son necesarios estudios multicéntricos y prospectivos para conocer mejor la fisiopatología, los criterios diagnósticos, el tratamiento y la existencia de complicaciones a medio y largo plazo.

Palabras clave: Infecciones por coronavírus; Inflamación; Pediatría.

\section{Introdução}

O SARS-CoV-2 (Severe Acute Respiratory Syndrome - CoV-2), foi detectado na cidade de Wuhan, na China, considerado agente etiológico da COVID-19. Esse patógeno é altamente infeccioso e logo se tornou uma emergência de saúde mundial. Sua transmissão se dá por meio do contato próximo com pessoas infectadas, tendo as gotículas respiratórias ou contato com objetos contaminados como principais veículos de transmissão, sendo as vias aéreas inferiores como o principal alvo da infecção (Calvo, López-Hortelano, Vicente \& Martínez, 2020).

A COVID-19 apresenta um variado espectro clínico e, quando presente, os sintomas principais são tosse, febre, coriza, dor de garganta, anosmia, ageusia, distúrbios gastrintestinais e astenia. Já em casos mais graves, há a instalação de um cenário de síndrome gripal, caracterizado pela presença de um quadro respiratório agudo, associado à pelo menos dois dos seguintes sintomas: sensação febril ou febre, dor de garganta, cefaleia, tosse e coriza (Hong, Wang, Chun \& Chen, 2020).

Ao contrário da população adulta, as crianças responderam a uma porção mínima dos casos na pandemia global da COVID-19. Em um estudo envolvendo 149.082 pacientes infectados pelo SARS-CoV-2 de idade conhecida, cerca de 2.572 $(1,7 \%)$ ocorreram em pacientes menores de 18 anos. Além disso, dados epidemiológicos de muitos países mostraram reduzido potencial de gravidade, pesquisas envolvendo 741 de pacientes pediátricos infectados pelo SARS-CoV-2 apresentaram sintomas leves $(50,9 \%)$, enquanto apenas 5,9\% estavam classificados como condição grave ou crítica (Diorio et al., 2020; Eastin \& Eastin, 2020).

Entretanto, nos últimos meses, os olhares dos centros de saúde voltaram-se para o público pediátrico, visto que, além 
da vulnerabilidade desses pacientes, foram identificadas crianças que desenvolveram uma resposta inflamatória sistêmica significativa, com características clínicas de uma doença grave, semelhante à doença de Kawasaki, associada à infecção por SARS-CoV-2, trazendo uma nova faceta dessa doença (Beroukhim \& Friedman, 2020).

Nesse contexto, devido ao elevado impacto global da COVID-19, bem como a relevante preocupação mediante ao surgimento de casos de Síndrome Inflamatória Pediátrica (SIM-P) temporariamente associada à COVID-19, esse estudo teve como objetivo analisar a literatura científica atual a respeito da SIM-P, com o intuito de fornecer informações relevantes e atuais para documentar os principais achados que associam a essa às infecções por SARS-CoV-2, bem como seu manejo terapêutico.

\section{Metodologia}

As revisões sistemáticas em saúde são fundamentais para reunir evidências atuais diretamente ligadas à segurança e eficácia das intervenções na área, viabilizando dados com exatidão, credibilidade, além de possibilitar ao leitor avaliar o assunto abordado, compreendendo os pontos positivos e negativos mediante análise. Nesse sentido, o presente estudo trata-se de uma revisão da literatura, de caráter qualitativo, elaborada conforme as instruções contidas no Preferred Reporting Items for Systematic reviews and Meta-Analyses (PRISMA) (Liberati et al., 2009).

A metodologia PRISMA emprega o uso de uma lista de verificação utilizada no processo de revisão objetivando aperfeiçoar os relatos encontrados por meio das revisões sistemáticas e metanálises. Nesta investigação, as bases eletrônicas de dados sistematicamente explanadas entre setembro de 2020 e fevereiro de 2021 foram Scientific Electronic Library Online (Scielo), National Library of Medicine (PubMed) e ScienceDirect (Liberati et al., 2009).

Todas as fases de elaboração do estudo, pesquisa, seleção, extração e análise dos dados foram realizadas aos pares, seguida de discussão entre os revisores, escolhendo os estudos pertinentes para o desenvolvimento da pesquisa. Na existência de qualquer discordância, um terceiro revisor foi consultado para deliberação e resolução.

Para a coleta de dados foi utilizada a técnica de documentos, que segundo Pereira et al. (2018), são procedimentos que auxiliam na operacionalização dos métodos, por meio da busca de artigos publicados em revistas e jornais que possam contribuir no resultado final da pesquisa.

Os artigos identificados foram examinados para determinar se descreviam pacientes com características clínicas e epidemiológicas consistentes com a Síndrome Inflamatória Multissistêmica Pediátrica (SIM-P) conforme os critérios definidos pelo Ministério da Saúde e Organização Mundial da Saúde (OMS).

Foram incluídos os artigos que apresentavam estudos observacionais com pacientes diagnosticados com SIM-P temporariamente associada à COVID-19, artigos originais e metanálises publicados entre os anos 2020 e 2021 . Os dados coletados dos documentos incluíram informações demográficas, sinais e sintomas clínicos e resultados, marcadores laboratoriais de inflamação, função cardiovascular e tratamentos administrados.

Foram excluídos estudos que não descreviam suficientemente os dados, como também aqueles que não apresentavam relação com objetivo tema desta revisão bibliográfica, notícias, editoriais, comentários e cartas de apresentação, assim como, as duplicatas.

As buscas em todas as bases eletrônicas de dados foram realizadas por meio da combinação dos descritores e palavras-chave, multisystem inflammatory syndrome, children e COVID-19, no idioma inglês, adicionados dos operadores booleanos AND e OR.

\section{Resultados e Discussão}

Desde a confirmação do primeiro caso de infecção pediátrica por SARS-CoV-2 registrado na cidade de Shenzhen, 
observa-se um aumento constante do número de crianças e adolescentes diagnosticados com COVID-19, onde em novembro de 2020, esse índice ultrapassou a marca de 1 milhão de casos. Dessa forma, a apresentação clínica e as características epidemiológicas da SARS-CoV-2 no público pediátrico são continuamente estudadas e novos dados surgem diariamente (Adeyinka, Bailey, Pierre \& Kondamudi, 2021).

A Figura 1 elucida as 618 publicações detectadas nas três bases eletrônicas de dados consultadas, das quais, após seleção e exclusão dos estudos duplicados, 201 trabalhos foram analisados, levando em consideração os critérios de inclusão e exclusão mencionados na metodologia, bem como a relevância das informações. Posteriormente a esse estágio, 57 pesquisas foram analisadas com a leitura total do texto e, ao final dessa apreciação, 27 artigos foram introduzidos nesta revisão.

Figura 1. Fluxograma demostrando o processo de seleção de artigos nas bases de dados utilizadas.

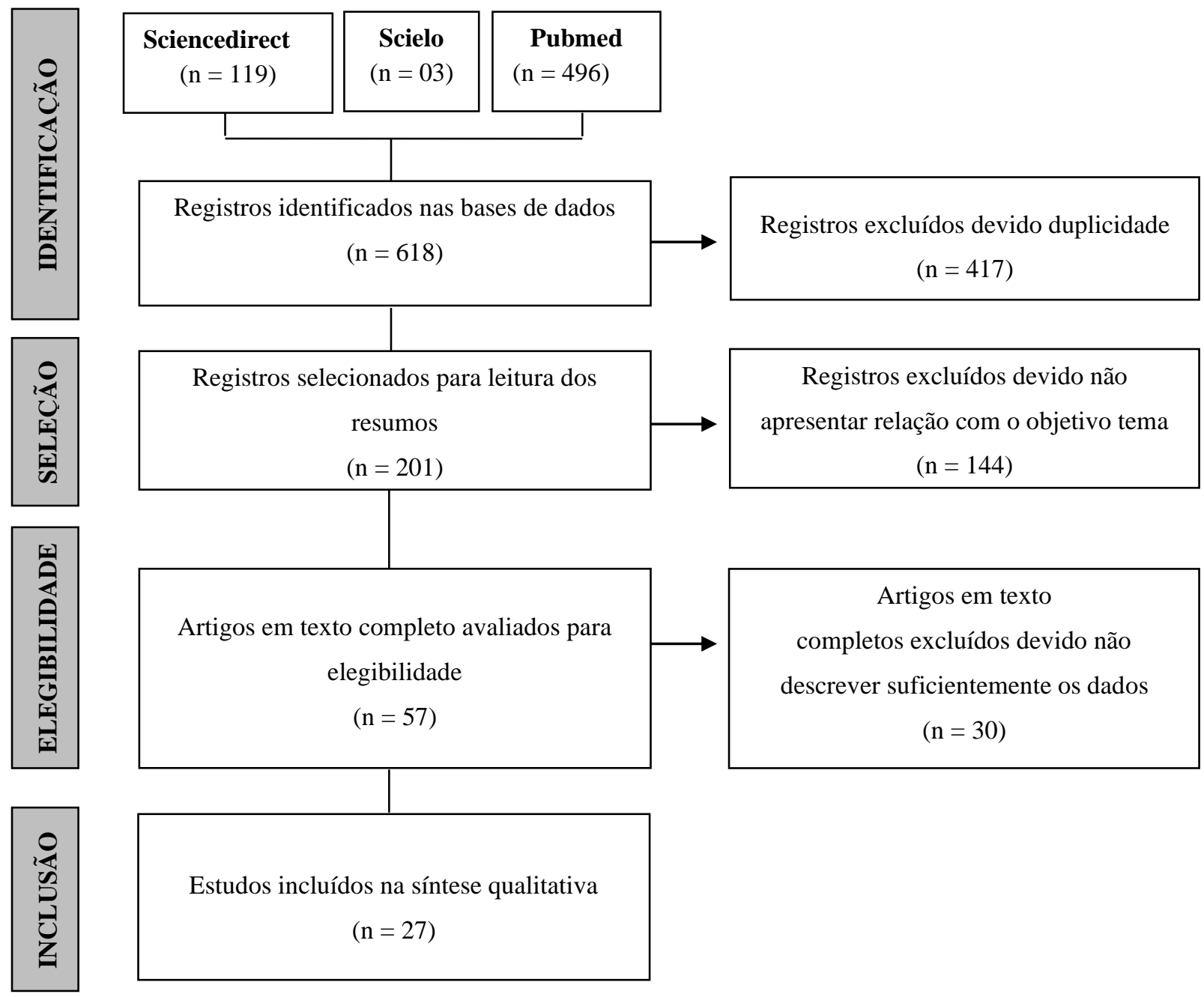

Fonte: Autores.

Diversas hipóteses trazem que as reduzidas taxas de infecção no público pediátrico estão relacionadas a leviandade dos casos, disponibilidade e validade dos testes atuais, menor expressão do receptor ACE2, melhor resposta imune inata, resposta pró-inflamatória com reduzida produção de citocinas e menor prevalência de comorbidades. Tais características, levam crianças acometidas por SARS-CoV-2 a apresentarem infecções leves ou assintomáticas (Song et al., 2020).

Quando presente, os sintomas são amplos e variáveis, no entanto, febre e tosse seca são as principais características clínicas descritas. Em uma análise retrospectiva com pacientes pediátricos, com média de idade de 8,3 anos, foi possível 
observar que os sintomas mais relatados foram temperatura $>37^{\circ} \mathrm{c}(45 \%)$ e tosse seca (24\%) e em menor quantidade cefaleia (10\%), vômito, diarreia ou dor de garganta (7\%), congestão nasal (3\%) e falta de ar (4\%) (Esposito \& Principi, 2021; Qiu et al., 2020).

De modo geral, o público pediátrico apresenta um bom prognóstico, com recuperação entre 1 ou 2 semanas e uma pequena parcela necessita de cuidados intensivos ou hospitalização. Estudos atuais apontam que lactentes (0-2 anos) e préescolares (2-4 anos) são mais susceptíveis a apresentar as manifestações clínicas graves do que aquelas crianças de idade mais avançada. Durante a avaliação da gravidade de 2.143 pacientes pediátricos com suspeita ou confirmados para COVID-19, foi possível constatar que a forma grave ou crítica da doença atingia $32 \%$ dos pacientes menores de 1 ano, e cerca de $28,8 \%$ de crianças entre 2 e 4 anos (Safadi, 2020).

À medida que o conhecimento sobre a COVID-19 evolui, novos aspectos sobre a doença surgem, e a visão que o curso da doença em crianças é sempre leve é agora contestada, visto que, nos últimos meses o número de relatos de crianças que apresentam quadro inflamatório com caráter multissistêmico temporariamente associada à infecção por SARS-CoV-2 vem surgindo, principalmente na Europa, América do Norte, Ásia e América Latina (Simon Junior et al., 2020; Ahmed et al., 2020).

\subsection{COVID-19 e a síndrome inflamatória multissistêmica em pacientes pediátricos}

Desde abril de 2020 os serviços de saúde de vários países publicaram alertas a respeito do surgimento de crianças gravemente enfermas apresentando choque hiperinflamatório com envolvimento de múltiplos órgãos e evidência sorológica de infecção pelo SARS-CoV-2. No Brasil, até o momento 646 crianças e adolescentes com faixa etária entre 0 a 19 anos foram diagnosticados com SIM-P temporariamente associada à COVID-19, e sua distribuição se dá por todo território nacional, com maior incidência nos estados de São Paulo (108), Ceará (64), Pará (63), Rio de Janeiro (56), Distrito Federal (48) e Bahia (47) (Ministério da Saúde, 2021, Janeiro).

A fisiopatologia da SIM-P não é clara, no entanto, estudos associam a uma resposta imunológica retardada ou uma má resposta adaptativa do hospedeiro mediada por citocinas a uma infecção recente por SARS-CoV-2 em vez de lesão viral direta. Durante a infecção das células dendríticas ou macrófagos pelo SARS-CoV-2 ocorre uma indução de reduzidos níveis de citocinas antivirais e elevação da síntese de citocinas inflamatórias, como o fator de necrose tumoral [TNF], interleucina [IL] 1, IL-6, IL-10 e interferon-y, que levam a expressão do fator tecidual, liberação de quininas inflamatórias e redução do número total de linfócitos, afetando a imunidade inata e adquirida. Assim, verifica-se uma desregulação excessiva da resposta imune, promovendo o desenvolvimento de hiperinflamação por meio da liberação desses mediadores (Jiang et al., 2020; Elias et al.,2020; Alunno, Carubbi \& Rodríguez-Carrio, 2020).

A SIM-P refere-se a uma doença de caráter multissistêmico, com uma diversidade de sinais e sintomas, incluindo febre persistente por três ou mais dias, associada a dor abdominal, conjuntivite, exantema, erupções cutâneas, edema de extremidades, hipotensão e disfunção de vários órgãos, como consequência das grandes quantidades de citocinas no organismo, além do extravasamento de fluidos e células do sistema imunológico, principalmente para o pulmão e coração (Guo et al., 2020).

As diferenças nos sinais e sintomas clínicos dos indivíduos com SIM-P e COVID-19 podem ser visualizadas no Gráfico 1, construído a partir da comparação de dois grandes estudos. 
Gráfico 1 - Comparação dos sinais e sintomas de indivíduos com SIM-P e COVID-19.

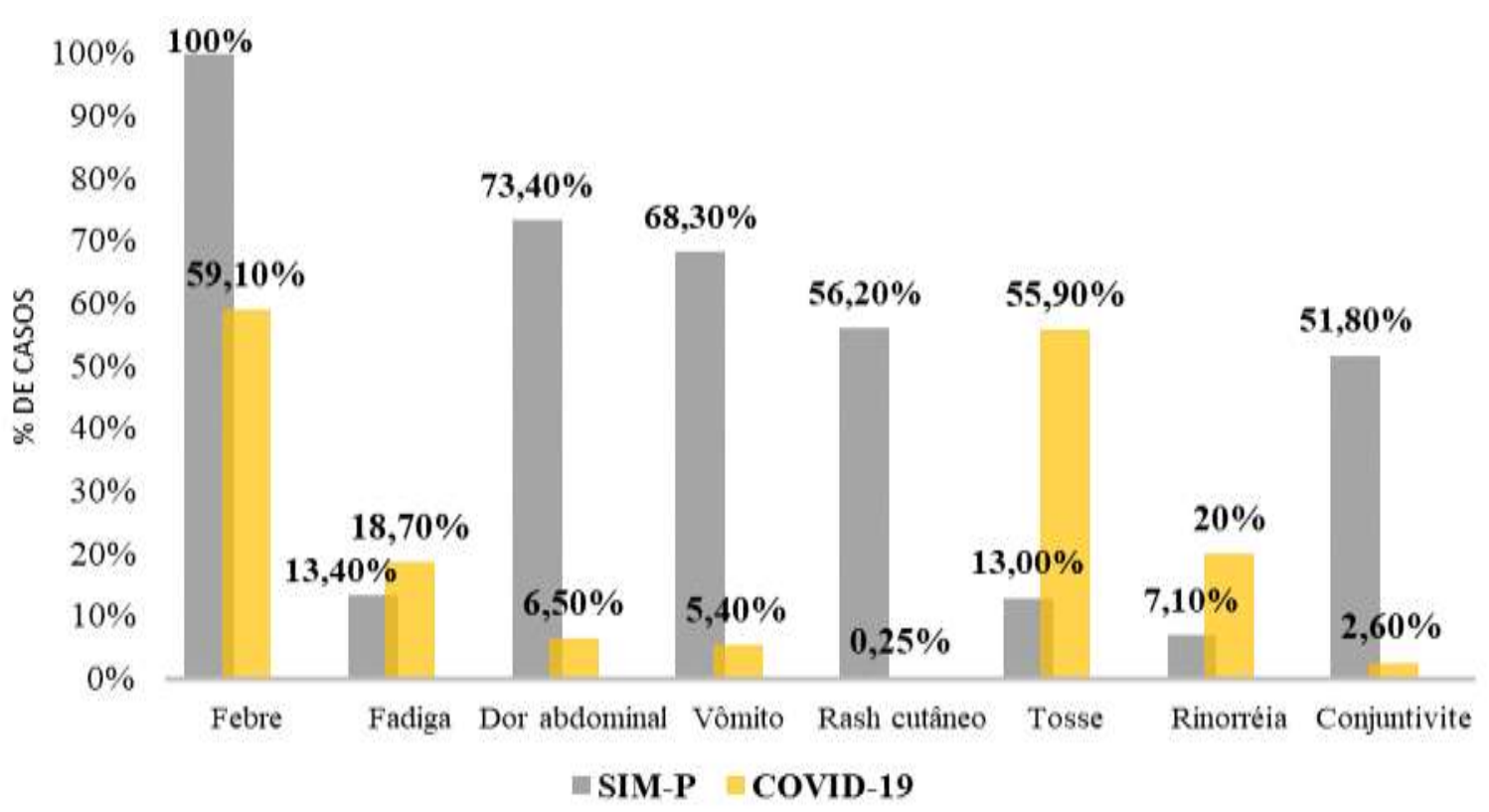

Fonte: adaptado de Ahmed et al. (2020); Hoang et al. (2020).

A sobreposição de apresentação dos sintomas pode ser apreciada, mas como principais diferenças na comparação de sinais e sintomas observa-se que febre, erupções cutâneas, vômitos, diarreia, dor abdominal e conjuntivite são mais comuns na SIM-P. Ao contrário, a COVID-19 apresenta um quadro clínico composto por sintomas respiratórios superiores, em especial tosse e rinorréia.

As características clínicas apresentadas pelo público pediátrico são continuamente estudadas mostrando variações de sinais e sintomas. Estudos envolvendo 58 crianças com média de idade de 9 anos foi realizado no intuito de descrever os aspectos clínicos dos pacientes que preencheram os critérios para SIM-P. 100\% dos pacientes apresentaram febre persistente por 3 a 19 dias, acompanhados de dor abdominal (53\%), erupções cutâneas eritematosas (52\%), conjuntivite (45\%), alterações da membrana mucosa e lábios (29\%), cefaleia (26\%), mãos e pés inchados (16\%) e dor de garganta (10\%) (Whittaker et al., 2020).

Compreender o curso clínico da SIM-P e sua associação temporal com a COVID-19 é de suma importância, dadas as implicações clínicas e de saúde pública da síndrome. Nesse sentido, caracterizar a epidemiologia, espectro da doença, curso clínico, tratamentos e prognóstico da SIM-P é a chave para reduzir a morbidade e mortalidade associada (Elias et al.,2020).

Um estudo com 186 pacientes pediátricos com média de idade de 8,3 anos diagnosticados com SIM-P, constatou que $70 \%$ desses tinham infecção por SARS-CoV-2 antecedente ou concomitante, enquanto os demais (30\%) tinham ligação epidemiológica com casos confirmados. Além disso, foi possível observar o envolvimento de múltiplos sistemas orgânicos, no qual, $71 \%$ dos pacientes apresentaram envolvimento de pelo menos quatro sistemas, sendo os mais relatados o gastrointestinal (92\%), cardiovascular (80\%), hematológico (76\%), mucocutâneo (74\%) e respiratório (70\%) (Feldstein et al., 2020).

A disfunção miocárdica é uma manifestação extrapulmonar proeminente de Covid-19 que também está sendo relatado nas crianças e adolescentes acometidos por SIM-P. Cerca de $80 \%$ dos casos podem apresentar um comprometimento vascular, particularmente aqueles pacientes que apresentem a forma grave da doença. Em um estudo com 58 crianças hospitalizadas com SIM-P, 50\% desenvolveram choque, frequentemente associado a evidências de disfunção ventricular esquerda na ecocardiografia $(n=18)$ arritmia $(n=9)$, bloqueio atrioventricular de primeiro grau com batimentos ectópicos supraventriculare s frequentes $(\mathrm{n}=1)$ e taquicardia de complexo amplo intratável $(\mathrm{n}=1)$ (Elias et al.,2020; Guo et al., 2020). 
Apesar da gravidade, pacientes diagnosticados com SIM-P apresentam bom prognóstico, com tempo de internação entre 4 e 10 dias. Vale salientar, que essa síndrome apresenta características patológicas com outras condições inflamatórias causadas por grandes quantidades de citocinas incluindo, doença de Kawasaki, sepse, síndrome de ativação macrofágica e linfo-histiocitose hemofagocítica secundária, o que por muitas vezes dificulta o seu diagnóstico (Alunno, Carubbi \& Rodríguez-Carrio, 2020).

\subsection{Achados laboratoriais}

Na presença de uma criança ou adolescente com quadro clínico sugestivo de SIM-P, diversos exames laboratoriais devem ser realizados para melhor investigação, os quais permitem uma averiguação mais aprofundada a respeito do acometimento de órgãos e sistemas, como também a monitorização da atividade inflamatória e do estado de hipercoagulabilidade (Feldstein et al., 2020).

Considerando que a SIM-P é uma manifestação clínica tardia a COVID-19, é importante uma verificação prévia de infecção por SARS-CoV-2. Esse resultado se dá por meio de sorologia positiva, com a presença de imunoglobulina G (IgG). Em estudos observacionais de pacientes que desenvolveram SIM-P mostram que a probabilidade de identificar a infecção por SARS-CoV-2 por sorologia foi cerca de duas a três vezes maior do que por RT-PCR, uma vez que, RT-PCR não apresenta uma sensibilidade clínica favorável na sua janela de positividade (Simon Junior et al., 2020; Prata-Barbosa et al., 2020).

Alterações laboratoriais são vistas nos indicadores inflamatórios como, elevação da proteína C-reativa, velocidade de hemossedimentação, procalcitonina, ferritina, alterações nos marcadores de coagulopatia como, tempo de protrombina, tempo de tromboplastina parcial ativado, dímero-D elevado, aumento das provas de função miocárdica como troponina, N-terminal do peptídeo natriurético tipo B (NT-proBNP), redução nos valores de linfócitos e albumina (Consiglio et al., 2020).

$\mathrm{Na}$ Tabela 1 encontram-se os principais achados laboratoriais vistos em uma série de estudos realizados em 37 pacientes pediátricos que se encontravam em processo investigativo para SIM-P.

Tabela 1 - Achados laboratoriais frequentemente encontrados em pacientes acometidos por SIM-P.

\begin{tabular}{cc}
\hline Achados laboratoriais & Número (\%) \\
\hline Linfopenia & $97 \%$ \\
\hline Proteína C reativa & $92 \%$ \\
\hline Dímero-D elevado & $91 \%$ \\
\hline Troponina & $90 \%$ \\
\hline Neutrófilos elevados & $89 \%$ \\
\hline Ferritina aumentada & $76 \%$ \\
\hline Albumina baixa & $74 \%$ \\
\hline Hemoglobina baixa & $68 \%$ \\
\hline
\end{tabular}

Fonte: adaptado de Viner \& Whittaker (2020).

Clinicamente, a elevação dos níveis de proteína C-reativa e ferritina observado na maioria dos casos caracteriza um estado hiperinflamatório, enquanto, o aumento do dímero-D e fibrinogênio pode-se associar à uma ativação da cascata de coagulação e presença de um estado pró-trombótico. Uma suspeita referente ao acometimento do sistema vascular é vista quando os níveis de Pro-BNP e troponina estão elevados.

Resultado semelhantes foram encontrados em um estudo realizado com 662 crianças com média de idade de 9,3 anos diagnosticadas com SIM-P. Nesse ensaio, 59,66\% dos pacientes apresentaram elevada contagem de leucócitos $\left(13,210^{3} / \mu \mathrm{L}\right)$, 
66,3\% tiveram alterações nas taxas de proteína C-reativa ( $160 \mathrm{mg} / \mathrm{L})$ e 46,22\% apresentavam linfopenia. Além disso, outros marcadores inflamatórios e cardíacos foram dosados e alterações nos níveis de peptídeo natriurético cerebral, ferritina e dímero-D (3.604 pg/mL, $303 \mathrm{ng} / \mathrm{mL}$ e 3,5mg/L respectivamente) foram encontrados, apresentando evidências de um estado inflamatório acentuado (Ahmed et al., 2020).

As alterações encontradas na proteína C-reativa, troponina, ferritina, NT-proBNP e a presença de neutrofilia e linfopenia são úteis como indicadores de gravidade da SIM-P. Estudos evidenciam que a piora clínica do paciente está relacionada a alterações laboratoriais significativas principalmente nos marcadores inflamatórios e enzimas cardíacas (Shah \& Munoz, 2020).

A utilização de exames de imagem é parte integrante para acompanhamento do paciente pediátrico acometido por SIM-P, pois permite o conhecimento das manifestações cardíacas, torácicas e extratorácicas. A literatura científica atual direciona que os pacientes pediátricos acometidos por SIM-P, apresentam achados de imagem semelhantes a síndrome do desconforto respiratório agudo, apresentando radiografias de tórax com opacidades em vidro fosco periféricas multifocais bilaterais com ou sem consolidações e distribuídos no lobo posterior e inferior (Sarzi-Puttini et al., 2020).

Devido cerca de 43 a 67\% dos pacientes acometidos por SIM-P apresentarem miocardite e insuficiência cardíaca, exames complementares como eletrocardiograma, ecocardiograma, angiotomografia computadorizada. As diretrizes da doença de Kawasaki devem ser utilizadas para acompanhamento dos pacientes, com a repetição de imagens em 1 a 2 semanas e 4 a 6 semanas após o tratamento para pacientes cujo curso da doença apresenta ecocardiografia simples e mais frequente para pacientes mais graves (Feldstein et al., 2020; Winant et al., 2020).

\subsection{Manejo terapêutico da SIM-P}

Os pacientes pediátricos acometidos por SIM-P podem evoluir para a forma grave da doença, com instalação de quadros de insuficiência respiratória aguda, hipotensão arterial, doença renal aguda, insuficiência cardíaca aguda e choque, necessitando de atenção da equipe pediátrica multiprofissional. Levantamentos apontaram que as especialidades envolvidas no manejo da SIM-P temporariamente associada ao COVID-16 incluem cardiologia (94\% internação e ambulatorial), infectologia (86\% internação, 47\% ambulatorial) e reumatologia (83\% internação, 69\% ambulatorial) (Elias et al.,2020; Sarzi-Puttini et al., 2020).

Até o momento não existe uma linha de tratamento completamente aceita, e os protocolos de gestão são direcionados de acordo com a gravidade do quadro clínico de cada paciente. Nesse sentido, além de cuidados gerais, medidas terapêuticas como imunoglobulina humana, corticosteroides, drogas vasoativas, imunomoduladores, anticoagulantes, antiplaquetários, antivirais e plasma convalescente, são necessários para reduzir a resposta inflamatória, reestabelecer as funções orgânicas e redução da mortalidade (Jiang et al., 2020; Simon Junior et al., 2020).

Observa-se uma semelhança nas condutas terapêuticas para o tratamento da SIM-P entre os centros de saúde. A Tabela 2 apresenta os resultados encontrados no tratamento de 662 pacientes pediátricos confirmados com SIM-P, onde terapia com imunoglobulina intravenosa foi o medicamento mais comum $(76,4 \%)$, seguido por agentes vasoativos e corticosteroides $(52,3 \%)$. 
Tabela 2 - Medicamentos utilizados em crianças com SIM-P.

\begin{tabular}{cc}
\hline Medicamento & Número de Pacientes (\%) \\
\hline Imunoglobulina humana & $76,4 \%$ \\
\hline Corticóides & $52,3 \%$ \\
\hline Suporte vasoativo / inotropismo positivo & $52,3 \%$ \\
\hline Anticoagulantes / antiplaquetários & $25,9 \%$ \\
\hline Antibióticos & $16,3 \%$ \\
\hline Inibidor de interleucina-1ra & $8,5 \%$ \\
\hline Inibidor de interleucina-6 & $6,0 \%$ \\
\hline Remdesivir & $0,9 \%$ \\
\hline Hidroxicloroquina & $0,8 \%$ \\
\hline
\end{tabular}

Fonte: adaptado de Ahmed et al. (2020).

Apesar de não existir um protocolo terapêutico definido, o uso das alternativas terapêuticas é pautado pela gravidade dos pacientes, como também, a eficácia demonstrada no tratamento de condições clínicas que apresentam um quadro inflamatório semelhante. Nesse sentido, o manejo da SIM-P em crianças e adolescentes deve ser de forma individualizada, levando em consideração a apresentação clínica, estado dos órgãos e os sistemas envolvidos

A imunoglobulina humana é amplamente utilizada no manejo de desordens imunológicas e inflamatórias. Dados apontam que esse agente deve ser administrado em doses de $2 \mathrm{~g} / \mathrm{kg}$, por meio de infusão prolongada em um período de 10 a 12 horas, nos pacientes que apresente a forma moderada ou e grave da doença, podendo ser fracionada em dois ou mais dias de acordo com as condições hemodinâmicas do paciente (Campos et al., 2020).

Estudos envolvendo 186 pacientes pediátricos (131 positivo para infecção por SARS-CoV-2, e 55 com ligação epidemiológica) internados em 53 hospitais, analisaram a farmacoterapia adotada em pacientes diagnosticados com SIM-P. O tratamento com imunoglobulina intravenosa foi a alternativa terapêutica mais utilizada nos dois grupos $100 \%$ e $97 \%$ respectivamente. Esse agente é de grande importância, visto que, sua administração pode ser combinada com antibióticos ou antivirais úteis para prevenir ou modificar infecções bacterianas e virais graves (Feldstein et al., 2020).

Os corticóides, em especial a metilprednisolona, integram cerca de 93\% dos protocolos atuais para o manejo da SIMP, especialmente em casos moderados ou graves, visto que, essa droga possui ação metabólica e anti-inflamatória potente. Esse medicamento é administrado em esquema de pulsoterapia concomitantemente a imunoglobulina, em doses que variam de acordo com a gravidade do paciente. Em casos leves doses de $2 \mathrm{mg} / \mathrm{kg} /$ dia dividida em 3 a 4 vezes são recomendadas. Já nos casos moderados e graves recomenda-se a administração de doses entre 10 a $30 \mathrm{mg} / \mathrm{kg} / \mathrm{dia}$ por 1 a 3 dias consecutivos, seguido de $2 \mathrm{mg} / \mathrm{kg} /$ dia por 5 dias, e desmame gradual ao longo de 2 a 3 semanas (Campos et al., 2020; Dove et al., 2020).

Em uma análise descritiva de 95 pacientes pediátricos acometidos por SIM-P foi possível observar que mais da metade dos pacientes (64\%) receberam glicocorticoides. Além do potencial anti-inflamatório, a associação de corticóides e imunoglobulina deve ser considerada mediante a possibilidade de comprometimento miocárdico, visto que, resultados positivos na redução do surgimento de aneurisma coronário foi constatado (Dufort et al., 2020).

Uma grande parte dos pacientes pediátricos acometidos por SIM-P apresentam disfunção ventricular e choque cardiogênico (67\%) e descompensação cardíaca aguda provocada pelo estado inflamatório grave (80\%), dessa forma, a utilização de fármacos inotrópicos como dobutamina ou milrinona são úteis para gerenciar o baixo débito cardíaco, hipoperfusão, insuficiência cardíaca e disfunção nos pacientes com pressão arterial sistêmica adequada. Na presença de hipotensão, a administração de epinefrina por infusão contínua foi a droga de escolha (Belhadjer et al., 2020; Winant et al., 
2020).

Outros medicamentos podem ser necessários no manejo da SIM-P, visto que, devido às características laboratoriais sugestivas a um estado pró-coagulável, o uso de terapias antiplaquetárias tem sido frequentemente relatado. Os protocolos sugerem a utilização de ácido acetil salicílico (AAS) quando o paciente apresentar manifestações da síndrome de Kawasaki e/ou plaquetose $(\geq 450.000 / \mu \mathrm{L})$, na dosagem de 30 a $50 \mathrm{mg} / \mathrm{kg} / \mathrm{dia}$, e dosagem máxima de $80 \mathrm{mg} /$ dia, sendo sua dose reduzida para 3 a $5 \mathrm{mg} / \mathrm{kg} /$ dia quando o paciente estiver afebril por 48 horas (Elias et al.,2020).

Já a utilização de anticoagulantes é recomendada para pacientes que se encontram em estado grave, com quadro inflamatório acentuado, níveis de dímeros $\mathrm{D}$ elevados e alta concentração de fibrinogênio. A escolha do agente anticoagulante deve ser realizada com auxílio de um hematologista pediátrico, de forma que a dose, duração do tratamento e a escolha do anticoagulante seja baseado nas condições clínicas do paciente e do seu estado pró-trombótico. Em um acompanhamento realizado com 186 pacientes hospitalizados por SIM-P, 47\% faziam terapia de anticoagulação tendo como alternativas terapêuticas a heparina, enoxaparina, bivalirudina, varfarina e argatrobam (Jiang et al., 2020; Whittaker et al., 2020).

Em virtude das características clínicas apresentadas pelo paciente como proteína C-reativa elevada e aumento dos níveis de neutrófilos, a administração de antibióticos não pode ser adiada devido à possibilidade de síndrome do choque tóxico. Dessa forma, a utilização de antibióticos de amplo espectro foi relatada devido às dificuldades em se excluir infecção bacteriana ou sepse. Estudos mostram que $86 \%$ dos pacientes pediátricos utilizaram antibióticos como cefalosporinas de terceira geração durante 6,5 dias enquanto se esperava por culturas bacterianas (Toubiana et al., 2020).

Em pacientes pediátricos com quadro clínico mais grave ou que apresentem resistência ao tratamento inicial com imunoglobulina e pulsoterapia com metilprednisolona opta-se pelo uso de agentes imunomoduladores como infliximabe (antifator de necrose tumoral), tocilizumab (antagonista de IL-6), e anakinra (antagonista do receptor de IL-1). Contudo, atualmente não se existe um consenso de qual alternativa é ideal no manejo da SIM-P, onde a escolha da alternativa terapêutica a ser utilizada vai depender dos resultados laboratoriais do paciente, principalmente do painel de citocinas (Feldstein et al., 2020).

A utilização de medicamentos antivirais como o remdesivir é limitado, visto que, a maioria dos pacientes acometidos por SIM-P não se encontram na fase aguda da doença. Assim, como essa droga atua inibindo a replicação viral ativa, seu uso é recomendado em casos raros, em que o teste de PCR é positivo e a criança está gravemente doente (Jiang et al., 2020).

\section{Conclusão}

O SARS-CoV-2 é um vírus novo e, atualmente, poucas evidências científicas estão disponíveis para compreender sua associação com a SIM-P. Essa síndrome apresenta sintomatologia e gravidade variável, podendo evoluir gravemente com falência múltipla de órgãos, necessitando de cuidados intensivos. Apesar de grandes avanços acerca dessa síndrome, inúmeras incertezas persistem, nesse sentido, estudos multicêntricos e prospectivos são necessários para possibilitar melhor entendimento dessa síndrome, seus mecanismos fisiopatológicos, critérios diagnósticos, estratégias de tratamento, assim como a existência de complicações de médio a longo prazo, tornando-se vertentes para futuros trabalhos.

\section{Referências}

Adeyinka, A., Bailey, K., Pierre, L., \& Kondamudi, N. (2021). COVID 19 infection: Pediatric perspectives. Journal of the American College of Emergency Physicians open, 2(1), e12375.

Ahmed, M., Advani, S., Moreira, A., Zoretic, S., Martinez, J., Chorath, K., Acosta, S., Naqvi, R., Burmeister-Morton, F., Burmeister, F., Tarriela, A., Petershack, M., Evans, M., Hoang, A., Rajasekaran, K., Ahuja, S., \& Moreira, A. (2020). Multisystem inflammatory syndrome in children: A systematic review. EClinicalMedicine, 26, 100527. 
Alunno, A., Carubbi, F., \& Rodríguez-Carrio, J. (2020). Storm, typhoon, cyclone or hurricane in patients with COVID-19? Beware of the same storm that has a different origin. RMD open, 6(1), e001295.

Belhadjer, Z., Méot, M., Bajolle, F., Khraiche, D., Legendre, A., Abakka, S., Auriau, J., Grimaud, M., Oualha, M., Beghetti, M., Wacker, J., Ovaert, C., Hascoet, S., Selegny, M., Malekzadeh-Milani, S., Maltret, A., Bosser, G., Giroux, N., Bonnemains, L., Bordet, J., \& Bonnet, D. (2020). Acute Heart Failure in Multisystem Inflammatory Syndrome in Children in the Context of Global SARS-CoV-2 Pandemic. Circulation, 142(5), 429-436.

Beroukhim, R. S., \& Friedman, K. G. (2020). Children at Risk: Multisystem Inflammatory Syndrome and COVID-19. JACC. Case reports, 2(9), 1271-1274.

Calvo, C., López-Hortelano, M. G., Vicente, J. C. D. C., \& Martínez, J. L. V. (2020). Recommendations on the clinical management of the COVID-19 infection by the «new coronavirus» SARS-CoV2. Spanish Paediatric Association working group. An Pediatr (Engl Ed), 92 (4), 241.e1-241.e11.

Campos, L. R., Cardoso, T. M., Martinez, J. C. F. F., Almeida, R. G., Silva, R. M., Fonseca, A. R., \& Sztajnbok, F. R. (2020). Pediatric inflammatory multisystem syndrome (PIMS) temporally related to SARS-CoV-2. Resid Pediatr, 10(2):1-6.

Consiglio, C. R., Cotugno, N., Sardh, F., Pou, C., Amodio, D., Rodriguez, L., Tan, Z., Zicari, S., Ruggiero, A., Pascucci, G. R., Santilli, V., Campbell, T., Bryceson, Y., Eriksson, D., Wang, J., Marchesi, A., Lakshmikanth, T., Campana, A., Villani, A., Rossi, P., \& Brodin, P. (2020). The Immunology of Multisystem Inflammatory Syndrome in Children with COVID-19. Cell, 183(4), 968-981.e7.

Diorio, C., Henrickson, S. E., Vella, L. A., McNerney, K. O., Chase, J., Burudpakdee, C., Lee, J. H., Jasen, C., Balamuth, F., Barrett, D. M., Banwell, B. L., Bernt, K. M., Blatz, A. M., Chiotos, K., Fisher, B. T., Fitzgerald, J. C., Gerber, J. S., Gollomp, K., Gray, C., Grupp, S. A., \& Bassiri, H. (2020). Multisystem inflammatory syndrome in children and COVID-19 are distinct presentations of SARS-CoV-2. The Journal of clinical investigation, $130(11)$, 5967-5975.

Dove, M. L., Jaggi, P., Kelleman, M., Abuali, M., Ang, J. Y., Ballan, W., Basu, S. K., Campbell, M. J., Chikkabyrappa, S. M., Choueiter, N. F., Clouser, K. N., Corwin, D., Edwards, A., Gertz, S. J., Ghassemzadeh, R., Jarrah, R. J., Katz, S. E., Knutson, S. M., Kuebler, J. D., Lighter, J., Mikesell, C., Mongkolrattanothai, K., Morton, T., Nakra, N. A., Olivero, R., Osborne, C. M., Panesar, L. E., Parsons, S., Patel, R. M., Schuette, J., Thacker, D., Tremoulet, A. H., Vidwan, N. K., \& Oster, M. E. (2020). Multisystem Inflammatory Syndrome in Children: Survey of Protocols for Early Hospital Evaluation and Management. J Pediatr. 2021 Feb: 229:33-40.

Dufort, E. M., Koumans, E. H., Chow, E. J., Rosenthal, E. M., Muse, A., Rowlands, J., Barranco, M. A., Maxted, A. M., Rosenberg, E. S., Easton, D., Udo, T., Kumar, J., Pulver, W., Smith, L., Hutton, B., Blog, D., \& Zucker, H. New York State and Centers for Disease Control and Prevention Multisystem Inflammatory Syndrome in Children Investigation Team. (2020) Multisystem Inflammatory Syndrome in Children in New York State. N Engl J Med. 2020 Jul 23;383(4):347-358.

Eastin, C., \& Eastin, T. (2020). Epidemiological characteristics of 2143 pediatric patients with 2019 coronavirus disease in China. The Journal of Emergency Medicine, 58(4), 712-713.

Elias, M. D., McCrindle, B. W., Larios, G., Choueiter, N. F., Dahdah, N., Harahsheh, A. S., Jain, S., Manlhiot, C., Portman, M. A., Raghuveer, G., Giglia, T. M., Dionne, A., \& of the International Kawasaki Disease Registry. (2020). Management of Multisystem Inflammatory Syndrome in Children Associated With COVID-19: A Survey From the International Kawasaki Disease Registry. CJC open, 2(6), 632-640.

Esposito, S., \& Principi, N. (2021). Multisystem Inflammatory Syndrome in Children Related to SARS-CoV-2. Paediatric drugs, 1-11. Advance online publication.

Feldstein, L. R., Rose, E. B., Horwitz, S. M., Collins, J. P., Newhams, M. M., Son, M., Newburger, J. W., Kleinman, L. C., Heidemann, S. M., Martin, A. A., Singh, A. R., Li, S., Tarquinio, K. M., Jaggi, P., Oster, M. E., Zackai, S. P., Gillen, J., Ratner, A. J., Walsh, R. F., \& Fitzgerald, J. C., CDC COVID-19 Response Team (2020). Multisystem Inflammatory Syndrome in U.S. Children and Adolescents. The New England journal of medicine, 383(4), 334-346.

Guo, Y. R., Cao, Q. D., Hong, Z. S., Tan, Y. Y., Chen, S. D., Jin, H. J., Tan, K. S., Wang, D. Y., \& Yan, Y. (2020). The origin, transmission and clinical therapies on coronavirus disease 2019 (COVID-19) outbreak - an update on the status. Military Medical Research, 7(1), 11.

Hoang, A., Chorath, K., Moreira, A., Evans, M., Burmeister-Morton, F., Burmeister F, Naqvi, R., Petershack, M., \& Moreira A. (2020). COVID-19 in 7780 pediatric patients: A systematic review. EClinicalMedicine, 24, 100433.

Hong, H., Wang, Y., Chung, H., \& Chen, C. (2020). Clinical characteristics of novel coronavirus disease 2019 (COVID-19) in newborns, infants and children. Pediatr Neonatol, 61 (2), 131-132.

Jiang, L., Tang, K., Levin, M., Irfan, O., Morris, S. K., \& Wilson, K. (2020). COVID-19 and multisystem inflammatory syndrome in children and adolescentes. The Lancet Infectious Diseases, 20(11), e276 - e288.

Liberati, A., Altman, D. G., Tetzlaff, J., Mulrow, C., Gotzsche, P. C., Ioannidis, J. P. A., Clarke, M., Devereaux, P. J., Kleijnen, J., \& Moher, D. (2009). The PRISMA statement for reporting systematic reviews and meta-analyses of studies that evaluate healthcare interventions: explanation and elaboration. BMJ, $339, \mathrm{~b} 2700$.

Ministério da Saúde, Secretaria de Vigilância em Saúde. (2021). Boletim epidemiológico. 52(03), 1-31.

Pereira A. S., Shitsuka, D. M., Parreira, F. J., \& Shitsuka, R. (2018). Metodologia da pesquisa científica. In Colusso, P. R., Construção e validação de instrumentos e técnicas de coleta de dados (pp. 42-43). Santa Maria: UFSM, NTE.

Prata-Barbosa, A., Lima-Setta, F., Santos, G. R. D., Lanziotti, V. S., de Castro, R. E. V., de Souza, D. C., Raymundo, C. E., de Oliveira, F. R. C., de Lima, L. F. P., Tonial, C. T., Colleti, J. Jr., Bellinat, A. P. N., Lorenzo, V. B., Zeitel, R. S., Pulcheri, L., Costa, F. C. M. D., La Torre, F. P. F., Figueiredo, E. A. D. N, Silva, T. P. D., Riveiro, P. M., Mota, I. C. F. D., Brandão, I. B., de Azevedo, Z. M. A., Gregory, S. C., Boedo, F. R. O., de Carvalho, R. N., Castro, N. A. A. S. R., Genu, D. H. S., Foronda, F. A.K., Cunha, A. J. L. A., \& de Magalhães-Barbosa, M. C., Brazilian Research Network in Pediatric Intensive Care, (BRnetPIC). (2020). Pediatric patients with COVID-19 admitted to intensive care units in Brazil: a prospective multicenter study. J Pediatr (Rio J), $96(5)$, $582-592$.

Qiu, H., Wu, J., Hong, L., Luo, Y., Song, Q., \& Chen, D. (2020). Clinical and epidemiological features of 36 children with coronavirus disease 2019 (COVID19) in Zhejiang, China: an observational cohort study. The Lancet Infectious Diseases, 20(6), 689-696. 
Research, Society and Development, v. 10, n. 3, e5710313020, 2021

(CC BY 4.0) | ISSN 2525-3409 | DOI: http://dx.doi.org/10.33448/rsd-v10i3.13020

Safadi, M. (2020). The intriguing features of COVID-19 in children and its impact on the pandemic. Jornal de pediatria, 96(3), 265-268.

Sarzi-Puttini, P., Giorgi, V., Sirotti, S., Marotto, D., Ardizzone, S., Rizzardini, G., Antinori, S., \& Galli, M. (2020). COVID-19, cytokines and immunosuppression: what can we learn from severe acute respiratory syndrome?. Clinical and experimental rheumatology, 38(2), 337-342.

Shah, S. K., \& Munoz, A. C. (2020). Multisystem Inflammatory Syndrome in Children in COVID-19 Pandemic. Indian journal of pediatrics, 87(9), 671-673.

Simon Junior, H., Sakano, T., Rodrigues, R. M., Eisencraft, A. P., Carvalho, V., Schvartsman, C., \& Reis, A. (2020). Multisystem inflammatory syndrome associated with COVID-19 from the pediatric emergency physician's point of view. Jornal de pediatria, S0021-7557(20)30203-5.

Song, Y., Liu, P., Shi, X. L., Chu, Y. L., Zhang, J., Xia, J., Gao, X. Z., Qu, T., \& Wang, M. Y. (2020). SARS-CoV-2 induced diarrhoea as onset symptom in patient with COVID-19. Gut, 69(6), 1143-1144.

Toubiana, J., Poirault, C., Corsia, A., Bajolle, F., Fourgeaud, J., Angoulvant, F., Debray, A., Basmaci, R., Salvador, E., Biscardi, S., Frange, P., Chalumeau, M., Casanova, J. L., Cohen, J. F., \& Allali, S. (2020). Kawasaki-like multisystem inflammatory syndrome in children during the covid-19 pandemic in Paris, France: prospective observational study. BMJ (Clinical research ed.), 369, m2094.

Viner, R. M., \& Whittaker, E. (2020). Kawasaki-like disease: emerging complication during the COVID-19 pandemic. Lancet (London, England), 395(10239), 1741-1743.

Whittaker, E., Bamford, A., Kenny, J., Kaforou, M., Jones, C. E., Shah, P., Ramnarayan, P., Fraisse, A., Miller, O., Davies, P., Kucera, F., Brierley, J., McDougall, M., Carter, M., Tremoulet, A., Shimizu, C., Herberg, J., Burns, J. C., Lyall, H., Levin, M., \& PIMS-TS Study Group and EUCLIDS and PERFORM Consortia (2020). Clinical Characteristics of 58 Children With a Pediatric Inflammatory Multisystem Syndrome Temporally Associated With SARS-CoV-2. JAMA, 324(3), 259-269.

Winant, A. J., Blumfield, E., Liszewski, M. C., Kurian, J., Foust, A., \& Lee, E. Y. (2020). Thoracic Imaging Findings of Multisystem Inflammatory Syndrome in Children (MIS-C) Associated with COVID-19: What Radiologists Need to Know Now. Radiology. Cardiothoracic Imaging, 2 (4), e200346. 